\title{
Compression of root systems and the $E$-sequence
}

\author{
Kevin Purbhoo * \\ Submitted: Aug 25, 2007; Accepted: Apr 22, 2008; Published: Sep 15, 2008 \\ Mathematics Subject Classification: 17B20
}

\begin{abstract}
We examine certain maps from root systems to vector spaces over finite fields. By choosing appropriate bases, the images of these maps can turn out to have nice combinatorial properties, which reflect the structure of the underlying root system. The main examples are $E_{6}$ and $E_{7}$.
\end{abstract}

\section{Introduction}

The primary goal of this paper is to provide a convenient way of visualising the root systems $E_{6}$ and $E_{7}$. There are two important relations on a root system that one might wish to have a good understanding of: the poset structure, in which $\alpha>\beta$ if $\alpha-\beta$ is a sum of positive roots, and the orthogonality structure, in which $\alpha \sim \beta$ if $\alpha$ and $\beta$ are orthogonal roots.

In our paper on cominuscule Schubert calculus, with Frank Sottile [8], we found that our examples required a good simultaneous understanding both these structures. This is easy enough to acquire for the root systems corresponding to the classical Lie groups. In $A_{n}$, for example, one can visualise the positive roots as the entries of an strictly upper triangular $(n+1) \times(n+1)$ matrix, where the $i j$ position represents the root $x_{i}-x_{j}$. Then $\alpha \geq \beta$ if and only if $\alpha$ is weakly right and weakly above $\beta$. Orthogonality is also straightforward in this picture: $\alpha$ and $\beta$ are non-orthogonal if there is some $i$ such that crossing out the $i^{\text {th }}$ row and the $i^{\text {th }}$ column succeeds in crossing out both $\alpha$ and $\beta$. Figure 1 shows the roots orthogonal to $x_{3}-x_{5}$ in $A_{5}$.

In type $E$, it is less obvious how to draw such a concrete picture. Separately the two structures have been well studied in the contexts of minuscule posets [7, 9, 11], and strongly regular graphs (see e.g. [1, 3, 4]). However, once one draws the Hasse diagram of the posets, the orthogonality structure suddenly becomes mysterious. Of course, one can always calculate which pairs of roots are orthogonal, but we would prefer a picture which allows us to do it instantly. Thus the main thrust of this paper is to get to Figures 4 and 6 ,

${ }^{*}$ Department of Combinatorics and Optimization, University of Waterloo, Waterloo, ON, N2L 3G1, Canada; kpurbhoo@math.uwaterloo.ca. 


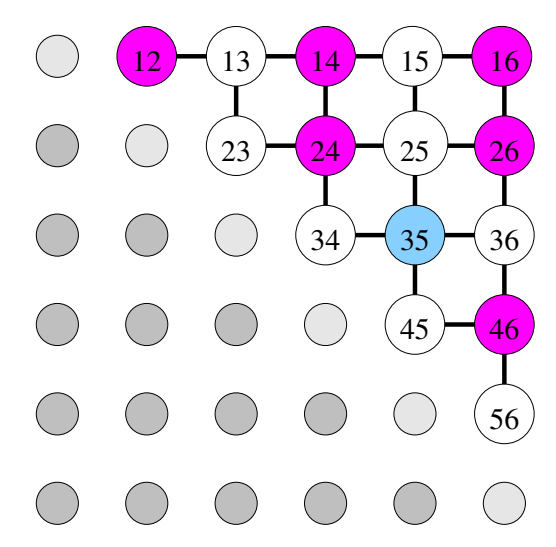

Figure 1: Orthogonality and partial order in type $A$.

which illustrate how one can simultaneously visualise $E_{7}$ and $E_{6}$ posets and orthogonality structures, at least restricted to certain strata of the root system. The restriction of these structures to the strata is exactly what is needed for the type $E$ examples in [8]. With a little more work, one can use these figures to recover the partial order and orthogonality structures for the complete root system.

To reach these diagrams, we begin by considering certain maps from a root system to $(\mathbb{Z} / p)^{m}$, which are injective (or $2: 1$ if $p=2$ ). In Section 2 , we make some general observations about these maps. Then, in Section 3 we give examples for $E_{6}$ and $E_{7}$ which are particularly nice. In these cases, we show that properties of the underlying root system are reflected in simple combinatorial structures on the target space, which is what allows us to produce diagrams in question. As the $E_{7}$ example is richer, we will discuss it before the $E_{6}$ example. Finally, in Section 4, we discuss how the partial order structures on each of the strata are related by order ideals. This relationship plays an essential role in [8], and is useful for understanding the structure of the $E_{8}$ root system.

The idea of relating the $E_{6}$ and $E_{7}$ root systems to $(\mathbb{Z} / p)^{m}$ has appeared elsewhere. For example, Harris [5] uses such an identification to describe the Galois group of the 27 lines on the cubic surface, one of the del Pezzo surfaces. The connection between del Pezzo surfaces and the exceptional Lie groups has been well established; we refer the reader to [6]. One can also see such a relationship reflected in the well known identification of Weyl groups (see e.g. [2]):

$$
\begin{gathered}
W\left(E_{6}\right) \cong S O(5 ; \mathbb{Z} / 3) \cong O^{-}(6 ; \mathbb{Z} / 2), \\
W\left(E_{7}\right) \cong \mathbb{Z} / 2 \times S p(6 ; \mathbb{Z} / 2) .
\end{gathered}
$$

These facts follow from the identifications outlined in this paper, and presumably have been proved in similar ways before. (To see that $W\left(E_{7}\right)$ identification is a direct product rather than a semidirect product, one needs the additional fact that the long word $w_{0} \in$ $W\left(E_{7}\right)$ is central.)

The author is grateful to Hugh Thomas and Richard Green for their comments on this paper. This work was partially supported by NSERC. 


\section{Compression of root systems}

\subsection{Simply laced root systems}

Let $\Delta \subset \mathbb{R}^{n}$ be a simply laced root system. Let $\langle\cdot, \cdot\rangle$ denote the inner product on $\mathbb{R}^{n}$, for which we have $\langle\beta, \beta\rangle=2$ for all $\beta \in \Delta$. We assume that $\Delta$ has full rank in $\mathbb{R}^{n}$. Let $\Lambda=\mathbb{Z} \Delta$ denote the lattice in $\mathbb{R}^{n}$ generated by $\Delta$.

Throughout, we will make use of the fact that if $\alpha$ and $\beta$ are roots in a simply laced root system $\Delta$, then $\alpha+\beta \in \Delta$ if and only if $\langle\alpha, \beta\rangle=-1$. Similarly, we have $\alpha-\beta \in \Delta$ if and only if $\langle\alpha, \beta\rangle=1$.

Choose a basis of simple roots $\alpha_{1}, \ldots, \alpha_{n} \in \Delta$, for $\Lambda$. Let $\Delta^{+}$denote the positive roots with respect to this basis, and $\Delta^{-}$denote the negative roots. Recall that $\Delta^{+}$is a partially ordered set, with $\beta>\beta^{\prime}$ iff $\beta-\beta^{\prime}$ is a sum of positive roots. Roots $\beta$ and $\beta^{\prime}$ are always comparable in the partial ordering when $\left\langle\beta, \beta^{\prime}\right\rangle>0$, though the converse is not true.

For each $\beta \in \Lambda$, we define $\beta^{i}$ to be the coefficient of $\alpha_{i}$, when $\beta$ is expressed in the basis of the simple roots: $\beta=\sum_{i=1}^{n} \beta^{i} \alpha_{i}$.

Let Dyn denote the Dynkin diagram of $\Delta$. As $\Delta$ is simply laced, each component of Dyn has type $A D E$. The vertices of Dyn are denoted $v_{1}, \ldots, v_{n}$, and correspond (respectively) to the simple roots $\alpha_{1}, \ldots, \alpha_{n}$. When $\Delta$ is a simple root system (i.e Dyn has just one component), the affine Dynkin diagram $\widehat{\text { Dyn }}$ is obtained by adding a vertex $\hat{v}_{n}$ to Dyn, corresponding to the lowest root $\hat{\alpha}_{n}$ of $\Delta$. Thus $-\hat{\alpha}_{n}$ is the highest root, and in particular is a positive root.

In addition to the usual named types $\left(A_{n}, D_{m}, m \geq 4, E_{6}, E_{7}, E_{8}\right)$, we will adopt the conventions that $D_{2}=A_{1} \times A_{1}, D_{3}=A_{3}, E_{3}=A_{2} \times A_{1}, E_{4}=A_{4}$, and $E_{5}=D_{5}$. (Note that on the level of root systems, the product is a disjoint union in the direct sum of the ambient vector spaces.)

\subsection{Root systems over $\mathbb{Z} / p$}

Let $p \geq 2$ be a positive integer. For reasons explained later in this section, the most interesting cases will be when $p$ is a prime, $p=4$, or $p=6$. Let $V$ be a finite rank free module over $\mathbb{Z} / p$, with a symmetric bilinear form $(\cdot \mid \cdot)$ taking values in $\mathbb{Z} / p$. Let $\Gamma=\{x \in V \backslash\{\overrightarrow{0}\} \mid(x \mid x)=2\}$.

Suppose that $\Gamma$ has a subset $S=\left\{a_{1}, \ldots, a_{n}\right\}$ such that

$$
\begin{aligned}
& \qquad\left(a_{i} \mid a_{j}\right)=\left\langle\alpha_{i}, \alpha_{j}\right\rangle \quad(\bmod p), \quad \text { for all } i, j \\
& \text { and if } p=2 \text { or } 3 \text { assume: } \quad a_{i} \neq a_{j}, \quad \text { for all } i \neq j \text {. }
\end{aligned}
$$

Then we obtain a map $f: \Lambda \rightarrow V$ by extending the natural map $\alpha_{i} \mapsto a_{i}$ to a homomorphism of abelian groups.

Proposition 2.2.1. If $\beta, \beta^{\prime} \in \Lambda$ then

$$
\left(f(\beta) \mid f\left(\beta^{\prime}\right)\right)=\left\langle\beta, \beta^{\prime}\right\rangle \quad(\bmod p)
$$


Proof. This is true for all pairs of simple roots, and both inner products are bilinear.

Corollary 2.2.2. Suppose $\beta \neq \pm \beta^{\prime} \in \Delta$. Then $\left\langle\beta, \beta^{\prime}\right\rangle=0$ if and only if $\left(f(\beta) \mid f\left(\beta^{\prime}\right)\right)=0$.

Proof. Since $\beta, \beta^{\prime}$ are roots of a simply laced root system, $\left\langle\beta, \beta^{\prime}\right\rangle \in\{-1,0,1\}$, thus $\left\langle\beta, \beta^{\prime}\right\rangle=0 \Longleftrightarrow\left\langle\beta, \beta^{\prime}\right\rangle=0(\bmod p) \Longleftrightarrow\left(f(\beta) \mid f\left(\beta^{\prime}\right)\right)=0$.

We now restrict the domain of $f$ to $\Delta$ if $p>2$ and to $\Delta^{+}$if $p=2$.

Theorem 2.2.3. If $p>2$, the map $f: \Delta \rightarrow V$ is injective, and its image lies in $\Gamma$. If $p=2$, the map $f: \Delta^{+} \rightarrow V$ is injective, and its image lies in $\Gamma$.

Proof. We first suppose $p>2$. Note that the fact that $f(\Delta) \subset \Gamma$ is clear from the fact that every $\beta \in \Delta$ satisfies $\langle\beta, \beta\rangle=2$.

Now, suppose that $\beta, \beta^{\prime} \in \Delta, f(\beta)=f\left(\beta^{\prime}\right)$. We show that $\beta=\beta^{\prime}$.

For all $\gamma \in \Delta$ we have $(f(\beta) \mid f(\gamma))=\left(f\left(\beta^{\prime}\right) \mid f(\gamma)\right)$, so $\langle\beta, \gamma\rangle=\left\langle\beta^{\prime}, \gamma\right\rangle(\bmod p)$. In particular the set of roots perpendicular to $\beta$ and $\beta^{\prime}$ are equal. This implies $\beta$ and $\beta^{\prime}$ belong to the same simple component of $\Delta$.

There are two cases: if the component is of type $A_{2}$, then it is easy to check that if $p \neq 3,(f(\beta) \mid f(\gamma))=\left(f\left(\beta^{\prime}\right) \mid f(\gamma)\right)$ for all $\gamma \in \Delta\left(A_{2}\right)$ implies $\beta=\beta^{\prime}$; if $p=3$ we need the additional hypothesis that $a_{i} \neq a_{j}$ for $i \neq j$ to draw the same conclusion. If the component is not of type $A_{2}$, then the fact that $\beta$ and $\beta^{\prime}$ have the same set of perpendicular roots implies that $\beta= \pm \beta^{\prime}$. (In types $D$ and $E$, the roots perpendicular to any given root span an entire hyperplane, and in type $A$ it is easily checked.) However, for all $x \in \Gamma, x \neq-x$. Since $f(\beta)=f\left(\beta^{\prime}\right) \in \Gamma$, we cannot have $\beta^{\prime}=-\beta$. Thus $\beta=\beta^{\prime}$.

For $p=2$, the fact that every $\beta \in \Delta^{+}$satisfies $\langle\beta, \beta\rangle=2$, implies that $f\left(\Delta^{+}\right) \subset$ $\Gamma \cup\{\overrightarrow{0}\}$. It is therefore enough to show that $f: \Delta^{+} \cup\{\overrightarrow{0}\} \rightarrow \Gamma \cup\{\overrightarrow{0}\}$ is injective.

Suppose $\beta, \beta^{\prime} \in \Delta^{+} \cup\{\overrightarrow{0}\}, f(\beta)=f\left(\beta^{\prime}\right)$. We show that $\beta=\beta^{\prime}$.

As in the $p>2$ case, for all $\gamma \in \Delta$, we have $\langle\beta, \gamma\rangle=\left\langle\beta^{\prime}, \gamma\right\rangle(\bmod 2)$. Thus the sets $P(\beta)$ and $P\left(\beta^{\prime}\right)$, where

$$
P(\beta):=\{\gamma \in \Delta \mid\langle\beta, \gamma\rangle=0\} \cup\{ \pm \beta\}
$$

coincide.

Note that $P(\beta)=\Delta$ if and only if $\beta=\overrightarrow{0}$ or $\beta$ belongs to an $A_{1}$ component. If $P(\beta)=P\left(\beta^{\prime}\right)=\Delta$, then $\beta$ and $\beta^{\prime}$ both belong to $A_{1}$ components, and hence are simple roots, or are zero; since $f$ restricted to $\left\{\overrightarrow{0}, \alpha_{1}, \ldots, \alpha_{n}\right\}$ is injective, we deduce $\beta=\beta^{\prime}$. So assume this is not the case. Then the elements of $\Delta \backslash P(\beta)$ belong to a single simple component of $\Delta$, namely the component containing $\beta$. Thus $\beta$ and $\beta^{\prime}$ belong to the same simple component of $\Delta$. Hence we may assume that $\Delta$ is a simple root system.

If $\Delta$ is of type $A_{2}$, then $P(\beta)=\{ \pm \beta\}$, hence $\beta=\beta^{\prime}$.

If $\Delta$ is of type $A_{k}, k \geq 4$, or of type $E_{6}, E_{7}$ or $E_{8}$, then $P(\beta)$ is a root system of type $A_{k-2} \times A_{1}, A_{5} \times A_{1}, D_{6} \times A_{1}$ or $E_{7} \times A_{1}$, respectively, where $\beta, \beta^{\prime}$ are both in the $A_{1}$ component. Thus in these cases $\beta=\beta^{\prime}$.

If $\Delta$ is of type $D_{k}, k \geq 3$ (including $D_{3}=A_{3}$ ), then $P(\beta)$ is a root system of type $D_{k-2} \times A_{1} \times A_{1}$, where $\beta, \beta^{\prime}$ are both in an $A_{1}$ component (a priori, not necessarily the 
same one). If $\beta, \beta^{\prime}$ belong to the same $A_{1}$ component, then $\beta=\beta^{\prime}$. So suppose they do not. The roots of $D_{k}$ are $\left\{e_{i} \pm e_{j} \mid i \neq j\right\} \subset \mathbb{R}^{k}$, for some orthonormal basis $e_{1}, \ldots, e_{k}$ of $\mathbb{R}^{k}$. It is easy to see that if $\beta=e_{i} \pm e_{j}$, then $\beta^{\prime}=e_{i} \mp e_{j}$. Now $f\left(2 e_{j}\right)=f(\beta)-f\left(\beta^{\prime}\right)=\overrightarrow{0}$; thus for all $l$, we have $f\left(2 e_{l}\right)=2 f\left(e_{l}-e_{j}\right)+f\left(2 e_{j}\right)=\overrightarrow{0}$. So $f\left(e_{m}+e_{l}\right)=f\left(e_{m}-e_{l}\right)$, for all $m \neq l$. But among these must be a pair of simple roots, namely the two simple roots conjugate under the Dynkin diagram automorphism. We conclude that $f$ restricted to the simple roots is not injective, contrary to (1).

Remark 2.2.4. Although we will not have use for it here, if $p$ is not a prime, one could also allow the possibility that $V$ is not a free module. In this case Theorem 2.2.3 remains true provided $f(\beta) \neq f(-\beta)$ for all $\beta \in \Delta$. This will be the case whenever $2 \nmid p$ or when Dyn has no component of type $A_{1}$.

We now show that the most interesting cases are when $p$ is a prime, $p=4$, or $p=6$. Suppose $p$ is composite, and not equal to 4,6 or 9 . Let $p^{\prime} \notin\{2,3\}$ be a proper divisor of $p$. Let $V^{\prime}=V \otimes_{\mathbb{Z} / p} \mathbb{Z} / p^{\prime}$. Let $\rho: V \rightarrow V^{\prime}$ denote the reduction modulo $p^{\prime}$ map. $V^{\prime}$ comes with a symmetric bilinear form $(\cdot \mid \cdot)^{\prime}$, the reduction of $(\cdot \mid \cdot)$ modulo $p^{\prime}$.

Corollary 2.2.5. The composite map $f^{\prime}:=\rho \circ f: \Delta \rightarrow V^{\prime}$ is injective, and its image lies in $\Gamma^{\prime}=\left\{x \in V^{\prime} \mid(x \mid x)^{\prime}=2\right\}$. Moreover $\left\langle\beta, \beta^{\prime}\right\rangle=\left(f^{\prime}(\beta) \mid f^{\prime}\left(\beta^{\prime}\right)\right)^{\prime}\left(\bmod p^{\prime}\right)$.

Proof. As $p^{\prime} \notin\{2,3\}$, we do not need the assumption that the $a_{i}$ are distinct; hence this follows from the fact that $\rho$ preserves inner products modulo $p^{\prime}$.

With some additional work, one can check that Corollary 2.2.5 is also true with $p=9$ and $p^{\prime}=3$.

\subsection{Compression}

The most interesting case of Theorem 2.2.3 occurs when rank $m$ of $V$ is smaller than the rank $n$ of $\Lambda$. If this is the case, we will call the map $f$ a compression of the root system. Here we give a necessary and nearly sufficient condition for compression to be possible.

Let $A$ be the Coxeter matrix of $\Delta, A_{i j}=\left\langle\alpha_{i}, \alpha_{j}\right\rangle$.

Proposition 2.3.1. If we have $S$ as in (1), and $m<n$, then $p \operatorname{divides} \operatorname{det}(A)$.

Proof. Let $\mathbf{s}$ be the $m \times n$ matrix whose columns are the $a_{i}$ in some basis, and let $g$ be the $m \times m$ matrix representing the bilinear form $(\cdot \mid \cdot)$ in the same basis. Then

$$
A_{i j}=\left(a_{i} \mid a_{j}\right)=\left(\mathbf{s}^{T} g \mathbf{s}\right)_{i j} \quad(\bmod p) .
$$

If $m<n$ then $\operatorname{det}\left(\mathbf{s}^{T} g \mathbf{s}\right)=0$, so $p \mid \operatorname{det}(A)$.

Conversely, if $p$ is prime and $p \mid \operatorname{det}(A)$, and $A_{p}$ denotes the reduction of $A$ modulo $p$, then one can define $V=(\mathbb{Z} / p)^{n} / \operatorname{ker}\left(A_{p}\right)$. Let $a_{i}$ is the image of the standard basis vector $e_{i}$ under the natural map. This will satisfy (1), provided the $a_{i}$ are all distinct and 
non-zero. The same construction works if $p$ is not prime, though $V$ will not necessarily be a free $\mathbb{Z} / p$-module.

In particular, we cannot hope for compression in $E_{8}$, a root system for which $\operatorname{det}(A)=$ 1. For $E_{7}$, however, $\operatorname{det}(A)=2$, and for $E_{6}$, $\operatorname{det}(A)=3$. Thus we should expect compression of the $E_{7}$ and $E_{6}$ root systems to be possible, taking $p=2$ or 3 respectively.

\subsection{Structures on $V$}

Define $\mathrm{O}(V)$ to be the graph whose vertex set is $V$ and whose edges are pairs $(x, y), x \neq y$ such that $(x \mid y)=0$. The graph $\mathrm{N}(V)$ is defined to be the complement of $\mathrm{O}(V)$, having vertex set $V$ and edges $(x, y)$ such that $(x \mid y) \neq 0$. If $X \subset V$, we denote the restrictions of $\mathrm{O}(V)$ and $\mathrm{N}(V)$ to $X$ by $\mathrm{O}(X)$ and $\mathrm{N}(X)$, respectively.

As our two main examples involve $p=2$ and $p=3$, we consider some special inner products $(\cdot \mid \cdot)$ in these case.

If $p=2$, we let $V$ be an even dimensional vector space over $\mathbb{Z} / 2$ with a symplectic form $(\cdot \mid \cdot)$. By symplectic form, we mean an (anti)symmetric non-degenerate bilinear form

for which $(x \mid x)=0$ for all $x$. Thus $\Gamma=V \backslash\{\overrightarrow{0}\}$. We see that $S \subset V \backslash\{\overrightarrow{0}\}$ satisfies the condition (1) iff the graph $\mathrm{N}(S)$ is isomorphic to Dyn. In this case, the associated map $f$ gives an injective map from $\Delta^{+}$to $V \backslash\{\overrightarrow{0}\}$.

If $p=3$, we take $V$ to be an $m$-dimensional vector space over $\mathbb{Z} / 3$, with the standard symmetric form

$$
\left(\left(x_{1}, \ldots, x_{m}\right) \mid\left(y_{1}, \ldots, y_{m}\right)\right)=\sum_{i=1}^{m} x_{i} y_{i}
$$

\section{Application to $E_{7}$ and $E_{6}$}

\subsection{The $E$-sequence}

Consider $\mathbb{R}^{8}$ with the standard Euclidean inner product $\langle\cdot, \cdot\rangle$. Let $\alpha_{1}, \ldots, \alpha_{8}$ be the vectors

$$
\begin{aligned}
& \alpha_{1}=(1,-1,0,0,0,0,0,0) \\
& \alpha_{2}=\left(\frac{1}{2}, \frac{1}{2}, \frac{1}{2},-\frac{1}{2},-\frac{1}{2},-\frac{1}{2},-\frac{1}{2},-\frac{1}{2}\right) \\
& \alpha_{3}=(0,1,-1,0,0,0,0,0) \\
& \alpha_{4}=(0,0,1,-1,0,0,0,0) \\
& \alpha_{5}=(0,0,0,1,-1,0,0,0) \\
& \alpha_{6}=(0,0,0,0,1,-1,0,0) \\
& \alpha_{7}=(0,0,0,0,0,1,-1,0) \\
& \alpha_{8}=(0,0,0,0,0,0,1,-1) .
\end{aligned}
$$

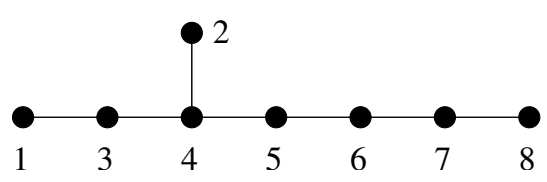

These are the simple roots of $E_{8}$, which span the $E_{8}$ lattice. They correspond to the vertices $v_{1}, \ldots, v_{8}$ of $\operatorname{Dyn}\left(E_{8}\right)$, in the order shown on the right. This ordering of simple 
roots of $E_{8}$ corresponds to the inclusion of root systems below.

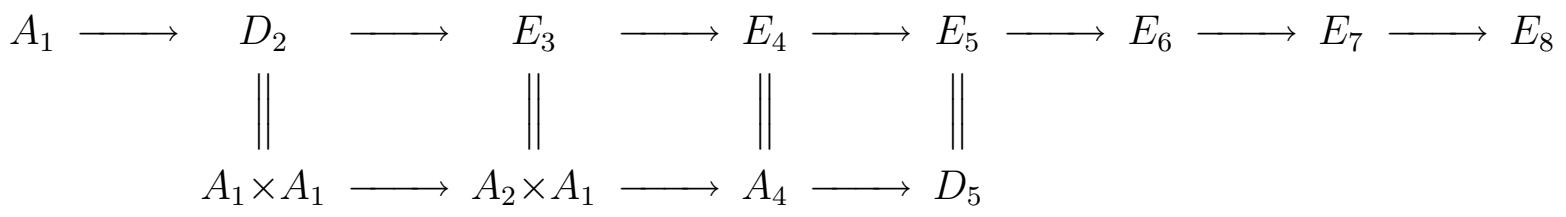

For $3 \leq n \leq 8$ the simple roots of $E_{n}$ are $\alpha_{1}, \ldots, \alpha_{n}$. These span the $E_{n}$ lattice $\Lambda\left(E_{n}\right)$. In general, the roots of $E_{n}$ are the lattice vectors $\alpha \in \Lambda\left(E_{n}\right)$ such that $\langle\alpha, \alpha\rangle=2$.

The positive roots $\Delta^{+}\left(E_{8}\right)$ of $E_{8}$ are stratified as $\Delta^{+}\left(E_{8}\right)=\amalg \Delta_{s}^{+}$. For $s \neq 2,3$,

$$
\Delta_{s}^{+}=\left\{\beta \in \Delta^{+}\left(E_{8}\right) \mid \beta \geq \alpha_{s} \text {, and } \beta \nsupseteq \alpha_{t} \text { for all } t>s\right\} .
$$

Equation (4) makes sense for $s=2$, and $s=3$; however it is convenient for our purposes (and arguably correct) to put these into the same stratum:

$$
\Delta_{3}^{+}=\left\{\alpha_{3}, \alpha_{3}+\alpha_{1}, \alpha_{2}\right\} .
$$

We also have a stratification of all roots of $E_{8}, \Delta\left(E_{8}\right)=\amalg \Delta_{s}$, where $\Delta_{s}=\Delta_{s}^{+} \cup-\Delta_{s}^{+}$. This stratification has the property that the roots of $E_{n}, 3 \leq n \leq 8$ are precisely

$$
\Delta\left(E_{n}\right)=\coprod_{s \leq n} \Delta_{s} .
$$

For notational convenience, we define $s^{\prime}=\max \{3, s+1\}$, so that $\Delta_{s}$ and $\Delta_{s^{\prime}}$ always denote consecutive strata.

For each stratum let $\mathrm{H}_{s}$ denote the graph whose vertices are $\Delta_{s}^{+}$and whose edges form the Hasse diagram of the poset structure on $\Delta^{+}$, restricted $\Delta_{s}^{+}$. Thus we have an edge joining $\beta$ and $\beta^{\prime}$ if one of $\pm\left(\beta-\beta^{\prime}\right)$ is a simple root. These are shown in Figure 2.

Finally, it is worth noting the size of each stratum. The stratification $\Delta^{+}\left(E_{8}\right)=\amalg \Delta_{s}^{+}$ has strata of sizes $1(s=1), 3(s=3), 6(s=4), 10(s=5), 16(s=6), 27(s=7)$ and $57(s=8)$.

\subsection{A compression of $E_{7}$}

We now take $\Delta$ to be the $E_{7}$ root system.

Let $F=(\mathbb{Z} / 2 \times \mathbb{Z} / 2, \oplus)$ denote the non-cyclic four element group. We denote the elements of this group $\{0,1,2,3\}$, and the operation $a \oplus b$ is binary addition without carry (also known as bitwise-xor). Thus $F$ is a two-dimensional vector space over $\mathbb{Z} / 2$ and thus hence admits a unique symplectic form:

$$
\left(a \mid a^{\prime}\right)= \begin{cases}0 & \text { if } a=0, a^{\prime}=0 \text { or } a=a^{\prime} \\ 1 & \text { otherwise }\end{cases}
$$

We shall take $V=F^{3}$, and whenever possible we write a triple $(a, b, c) \in V$ simply as $a b c$. We endow $V$ with the symplectic form

$$
\left(a b c \mid a^{\prime} b^{\prime} c^{\prime}\right)=\left(a \mid a^{\prime}\right)+\left(b \mid b^{\prime}\right)+\left(c \mid c^{\prime}\right) .
$$




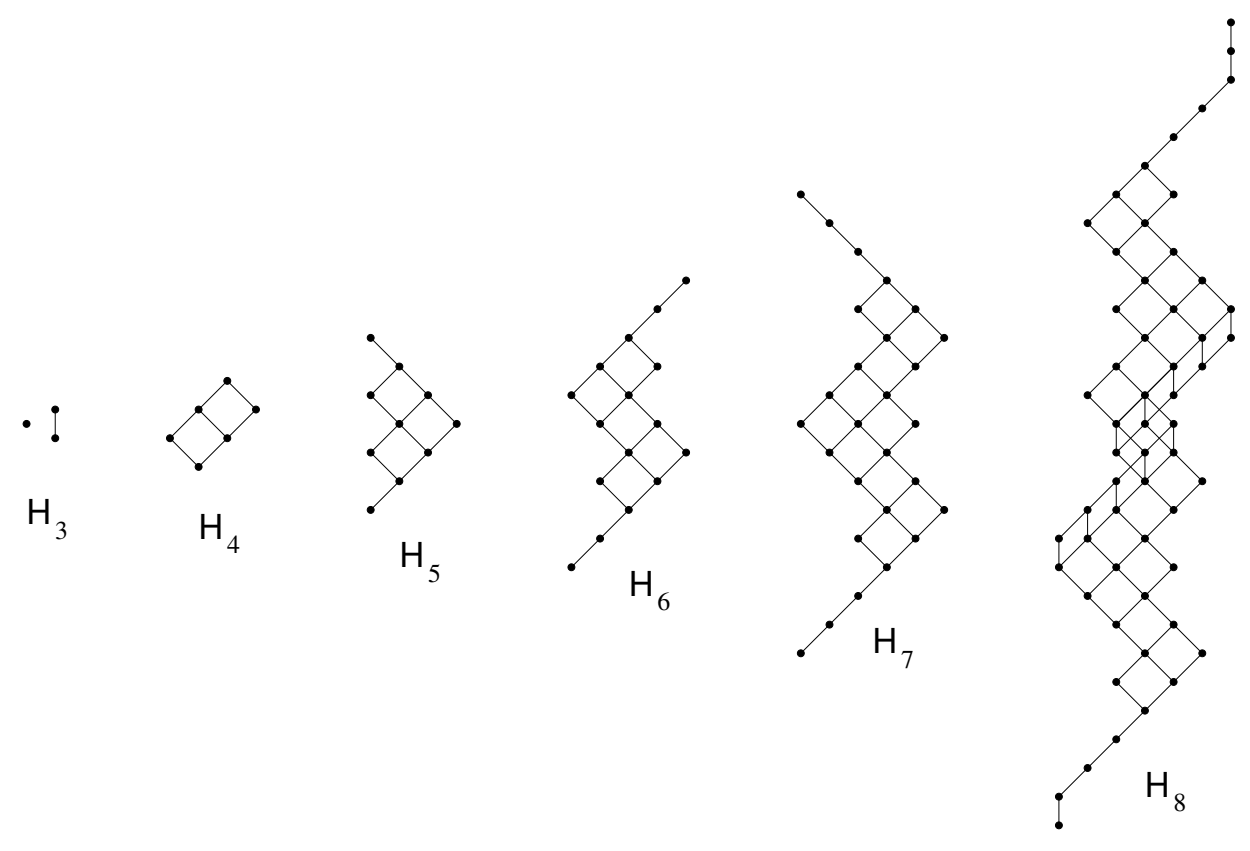

Figure 2: The Hasse diagrams $\mathrm{H}_{s}, 3 \leq s \leq 8$.

We take as our subset $S \subset V$, the set $S=\left\{a_{1}, \ldots, a_{7}\right\}$, where

$$
\begin{gathered}
a_{1}=100, \quad a_{2}=030, \\
a_{3}=300, \quad a_{4}=111, \\
a_{5}=003, \quad a_{6}=001, \quad a_{7}=033 .
\end{gathered}
$$

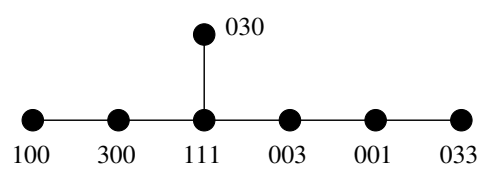

Proposition 3.2.1. The graph $\mathrm{N}(S)$ is $\operatorname{Dyn}\left(E_{7}\right)$. The natural homomorphism $f$ takes $\alpha_{i}$ to $a_{i}$.

Proof. This just needs to be checked.

As a consequence of we obtain the following corollary of Theorem 2.2.3.

Corollary 3.2.2. The map $f: \Delta^{+} \cup\{\overrightarrow{0}\} \rightarrow V$ is a bijection.

Proof. It is an injection by Theorem 2.2.3. But $\#\left(\Delta^{+} \cup\{\overrightarrow{0}\}\right)=\#(V)=64$, thus it is a bijection.

\subsection{Restriction to strata}

Let $\Gamma_{s}$ denote the image of the stratum $\Delta_{s}^{+}$under $f$. Here we show how natural structures on $\Delta_{s}^{+}$are preserved under $f$, and are more palatable in $\Gamma_{s}$.

We define a new graph structure on $V$. Let $\mathrm{T}(V)$ be the graph with vertex set $V=F^{3}$, and $a b c$ adjacent to $a^{\prime} b^{\prime} c^{\prime}$, if exactly one of $\left\{a=a^{\prime}, b=b^{\prime}, c=c^{\prime}\right\}$ holds. For $X \subset V$, let $\mathrm{T}(X)$ denote the restriction of $\mathrm{T}(V)$ to $X$. 
Unlike $\mathrm{O}(V)$, the graph $\mathrm{T}(V)$ has translation symmetries: for any $x \in V$, the map $y \mapsto x \oplus y$ is an automorphism. It is a strongly regular graph. In particular every vertex has valence 27 .

Definition 3.3.1. For $v \in V$, the link on $v$ in the $\boldsymbol{T}(V)$, denoted $\mathcal{L}(v)$, is the set of vertices of $\mathbf{T}(V)$ that are adjacent to $v$. Let $\mathcal{L}^{c}(v)$ denote the set of vertices of $\mathbf{T}(V)$ that are non-adjacent to $v$, excluding $v$ itself.

Lemma 3.3.2. The image of the largest stratum $\Gamma_{7}$ is $\mathcal{L}(\overrightarrow{0})$.

In other words, $\Gamma_{7}$ is the set of $a b c \in V$ such that exactly one of $\{a=0, b=0, c=0\}$ holds.

Note that this result is not independent of the choice of $S$ for the images of the simple roots. We have chosen $S$ quite carefully, in part to make this lemma hold. It is possible (and not difficult) to check this result on each of the 27 roots of $\Delta_{7}^{+}$; however, since a symmetry argument is available, we present it here.

Proof. We know that $f\left(\alpha_{7}\right)=033 \in \Gamma_{7}$, thus it suffices to show that $\Gamma_{7}$ is invariant under the following symmetries:

$$
\begin{aligned}
(a, b, c) & \mapsto([2 \leftrightarrow 3] \cdot a, b, c) & (a, b, c) & \mapsto([1 \leftrightarrow 2] \cdot a, b, c) \\
(a, b, c) & \mapsto(c, b, a) & (a, b, c) & \mapsto(a, c, b)
\end{aligned}
$$

The first two symmetries (5) are just the reflections in the simple roots $v_{1}$ and $v_{2}$ respectively, which are automorphisms of $\Delta_{7}^{+}$(c.f. Section 3.5). The second two symmetries (6) come from a Dynkin diagram construction, which we first describe for any $E_{n}$. A similar construction can also be used for types $A$ and $D$.

Let $\mathrm{D}=\operatorname{Dyn}\left(E_{n}\right)$. We decorate each vertex of $\mathrm{D}$ with the corresponding simple root in $\Delta$. Choose a vertex $v_{i} \in \mathrm{D}$, where $i \notin\{1,2,8\}$. If we delete the edge $\left(v_{i}, v_{i+1}\right)$ from $\mathrm{D}$, the diagram breaks up into two components $\mathrm{D}^{\prime}, \mathrm{D}^{\prime \prime}$ where $\mathrm{D}^{\prime}$ is the component containing $v_{1}$. If $i=n, \mathrm{D}^{\prime \prime}$ will be empty. Note that $\mathrm{D}^{\prime}$ is a sub-Dynkin diagram of $\mathbf{D}$, and hence corresponds to a sub-root system $\Delta^{\prime} \subset \Delta$.

We apply the following construction to obtain a new Dynkin diagram $\tilde{D}$ :

1. Add to $\mathrm{D}^{\prime}$ the affine vertex $\hat{v}_{i}$, to form the affine Dynkin diagram $\widehat{\mathrm{D}}^{\prime}$. This vertex is decorated with the lowest root $\hat{\alpha}_{i} \in \Delta^{\prime}$.

2. For every vertex $\widehat{\mathrm{D}^{\prime}}$, replace the root which decorates the vertex by its negative.

3. Delete the vertex $v_{i}$.

4. If $\mathrm{D}^{\prime \prime}$ is not empty, reattach it by forming an edge $\left(\hat{v}_{i}, v_{i+1}\right)$. The result is $\tilde{\mathrm{D}}$.

The underlying graph $\tilde{D}$ is isomorphic to $D$ : we identify the graphs $D$ and $\tilde{D}$ in such a way that $\mathrm{D}^{\prime \prime}$ is fixed, and $v_{i} \in \mathrm{D}$ corresponds to $\hat{v}_{i} \in \tilde{\mathrm{D}}$. Under this isomorphism, the roots decorating the vertices will have changed. Furthermore, the construction of $\tilde{D}$ respects the fact that the edges in the Dynkin diagram represent the inner products of the 
roots decorating the vertices; hence the roots decorating $\tilde{D}$ correspond to a new system of simple roots for $\Delta$. Thus this process corresponds gives an automorphism of $\Delta$, and hence to an automorphism of $\Gamma$.

Returning now to the $E_{7}$ case, we note each of these automorphisms is actually an extension of an automorphism of $\Delta\left(E_{6}\right)$. For $i \leq 6$ this is clear, and for $i=7$ it is the outer automorphism of $\Delta\left(E_{6}\right)$, given by reflecting the Dynkin diagram (Figure 3 (left)). Thus each automorphism restricts to an automorphism of $\Delta_{7}$, and hence of $\Gamma_{7}=f\left(\Delta_{7}^{+}\right)=$ $f\left(\Delta_{7}\right)$. The symmetries (6) are the automorphisms of $\Gamma_{7}$ given by the construction above, using vertices $v_{7}$ and $v_{6}$ respectively. It is sufficient to check this on the images of the simple roots. See Figure 3.
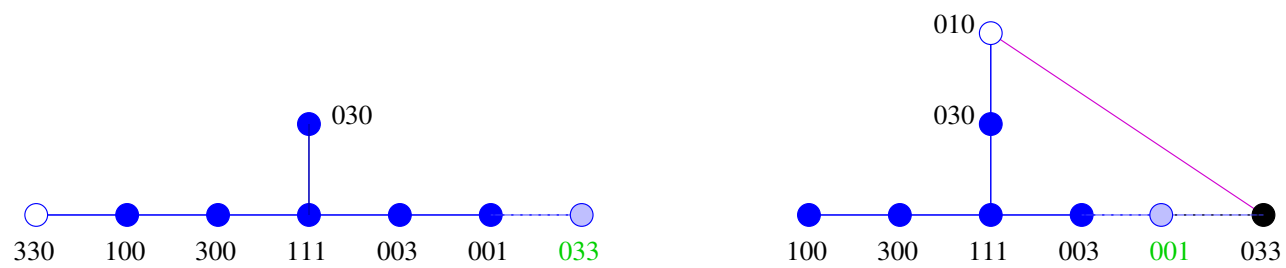

Figure 3: The automorphisms of $\Gamma_{7}$ described in the proof of Lemma 3.3.2, for $v_{7}$ (left) and $v_{6}$ (right).

Theorem 3.3.3. The graphs $\mathrm{T}\left(\Gamma_{7}\right)$ and $\mathrm{O}\left(\Gamma_{7}\right)$ coincide. Furthermore, the graphs $\mathrm{T}\left(\Gamma \backslash \Gamma_{7}\right)$ and $\mathrm{O}\left(\Gamma \backslash \Gamma_{7}\right)$ coincide. Thus, if either $\beta, \beta^{\prime} \in \Delta_{7}$, or $\beta, \beta^{\prime} \in \Delta\left(E_{6}\right)$, we have $\left\langle\beta, \beta^{\prime}\right\rangle=0$ if and only if $f(\beta)$ and $f\left(\beta^{\prime}\right)$ agree in exactly one coordinate.

Proof. We have $\left(a b c \mid a^{\prime} b^{\prime} c^{\prime}\right)=\left(a \mid a^{\prime}\right)+\left(b \mid b^{\prime}\right)+\left(c \mid c^{\prime}\right)$. Thus $\left(a b c \mid a^{\prime} b^{\prime} c^{\prime}\right)=0 \Longleftrightarrow$ an odd number of $\left\{\left(a \mid a^{\prime}\right),\left(b \mid b^{\prime}\right),\left(c \mid c^{\prime}\right)\right\}$ are zero. Write $a b c \sim a^{\prime} b^{\prime} c^{\prime}$ if $a b c$ and $a^{\prime} b^{\prime} c^{\prime}$ agree in exactly one coordinate.

First, let $a b c$ and $a^{\prime} b^{\prime} c^{\prime}$ be distinct elements of $\Gamma_{7}$. By Lemma 3.3.2 exactly one of $\{a, b, c\}$ and exactly one of $\left\{a^{\prime}, b^{\prime}, c^{\prime}\right\}$ is zero. Suppose $a=a^{\prime}=0$. Then we have $\left(a b c \mid a^{\prime} b^{\prime} c^{\prime}\right)=0 \Longleftrightarrow b \neq b^{\prime}$ and $c \neq c^{\prime} \Longleftrightarrow a b c \sim a^{\prime} b^{\prime} c^{\prime}$. Suppose $a=b^{\prime}=0$. Then $\left(a b c \mid a^{\prime} b^{\prime} c^{\prime}\right)=0 \Longleftrightarrow c=c^{\prime} \Longleftrightarrow a b c \sim a^{\prime} b^{\prime} c^{\prime}$. The remaining cases are the same by symmetry.

Now, let $a b c$ and $a^{\prime} b^{\prime} c^{\prime}$ be distinct elements of $\Gamma \backslash \Gamma_{7}$. By Lemma 3.3.2 an even number of $\{a, b, c\}$ are zero, and an even number of $\left\{a^{\prime}, b^{\prime}, c^{\prime}\right\}$ are zero. Suppose $a, b, c, a^{\prime}, b^{\prime}, c^{\prime}$ are all nonzero. Then $\left(a b c \mid a^{\prime} b^{\prime} c^{\prime}\right)=0 \Longleftrightarrow a b c$ and $a^{\prime} b^{\prime} c^{\prime}$ disagree in an even number of coordinates $\Longleftrightarrow a b c \sim a^{\prime} b^{\prime} c^{\prime}$. Suppose $a, b, c, a^{\prime}$ are nonzero and $b^{\prime}=c^{\prime}=0 .\left(a b c \mid a^{\prime} b^{\prime} c^{\prime}\right)=$ $0 \Longleftrightarrow a=a^{\prime} \Longleftrightarrow a b c \sim a^{\prime} b^{\prime} c^{\prime}$. Suppose $a, a^{\prime}$ are nonzero and $b=c=b^{\prime}=c^{\prime}=0$. Then $\left(a b c \mid a^{\prime} b^{\prime} c\right) \neq 0$ and $a b c \nsim a^{\prime} b^{\prime} c^{\prime}$. Suppose $a, b^{\prime}$ are nonzero and $b=c=a^{\prime}=c^{\prime}=0$. Then $\left(a b c \mid a^{\prime} b^{\prime} c\right)=0$ and $a b c \sim a^{\prime} b^{\prime} c^{\prime}$. The remaining cases are the same by symmetry.

The following construction provides a useful way of relating the other strata to $\Delta_{7}^{+}$. Put $z_{7}=\overrightarrow{0}, \zeta_{s}=\sum_{i=s^{\prime}}^{7} \alpha_{i}$, and $z_{s}=f\left(\zeta_{s}\right)$ for $s=1,3,4,5,6$. If $\beta \in \Delta_{s}^{+}$, define $\tilde{\beta}=\beta+\zeta_{s}$. 
Proposition 3.3.4. If $\beta, \beta^{\prime} \in \Delta_{s}^{+}$, then

(i) $\left\langle\beta, \zeta_{s}\right\rangle=-1$, and $\left\langle\beta, \zeta_{t}\right\rangle=0$, for $t>s^{\prime}$;

(ii) $\tilde{\beta}, \tilde{\beta}^{\prime} \in \Delta_{7}^{+}$;

(iii) $\left\langle\beta, \beta^{\prime}\right\rangle=\left\langle\tilde{\beta}, \tilde{\beta}^{\prime}\right\rangle$;

(iv) $\beta<\beta^{\prime}$ if and only if $\tilde{\beta}<\tilde{\beta}^{\prime}$.

Proof. We have $\left\langle\beta, \zeta_{s}\right\rangle=\left\langle\sum_{j=1}^{s} \beta^{j} \alpha_{j}, \sum_{i=s^{\prime}}^{7} \alpha_{i}\right\rangle=\left\langle\beta^{s} \alpha_{s}, \alpha_{s^{\prime}}\right\rangle=-\beta^{s}=-1$; similarly for $t>s^{\prime},\left\langle\beta, \zeta_{t}\right\rangle=\left\langle\sum_{j=1}^{s} \beta^{j} \alpha_{j}, \sum_{i=t}^{7} \alpha_{i}\right\rangle=0$; this proves (i). Thus we see that $\tilde{\beta}=\beta+\zeta_{s}$ is a root, and since $\tilde{\beta}^{7}=\beta^{7}+\zeta_{s}^{7}=1$, (ii) follows. We also deduce (iii), since $\left\langle\tilde{\beta}, \tilde{\beta}^{\prime}\right\rangle=$ $\left\langle\beta, \beta^{\prime}\right\rangle+\left\langle\beta, \zeta_{s}\right\rangle+\left\langle\zeta_{s}, \beta^{\prime}\right\rangle+\left\langle\zeta_{s}, \zeta_{s}\right\rangle=\left\langle\beta, \beta^{\prime}\right\rangle+(-1)+(-1)+2=\left\langle\beta, \beta^{\prime}\right\rangle$. Finally, (iv) follows from the fact that $\tilde{\beta}-\tilde{\beta}^{\prime}=\beta-\beta^{\prime}$.

In general, all of the strata can be described in terms of links in $\mathrm{T}(V)$. In view of Proposition 3.3.4, we also describe $\Gamma_{s} \oplus z_{s}=\left\{f(\tilde{\beta}) \mid \beta \in \Delta_{s}^{+}\right\}$, which is often easier to work with.

Theorem 3.3.5. We have the following identifications:

(i) $\Gamma_{s}=\mathcal{L}\left(z_{s}\right) \cap\left(\bigcap_{7 \geq t>s} \mathcal{L}^{c}\left(z_{t}\right)\right)$;

(ii) $\Gamma_{s} \oplus z_{s}=\mathcal{L}(\overrightarrow{0}) \cap\left(\bigcap_{7>t \geq s} \mathcal{L}^{c}\left(z_{t}\right)\right)$.

In particular, for $s \leq 6, \Gamma_{s} \oplus z_{s}=\left(\Gamma_{s^{\prime}} \oplus z_{s^{\prime}}\right) \cap \mathcal{L}^{c}\left(z_{s}\right)$. It is a pleasant fact, which the reader can check, that $z_{s}$ is always a minimal element (unique for $s \neq 1$ ) in the partial order in $z_{s^{\prime}} \oplus \Gamma_{s^{\prime}}$.

Proof. We already know this is true for $s=7$, so assume $s \leq 6$.

First, we calculate $\mathcal{L}(\overrightarrow{0}) \cap \mathcal{L}^{c}\left(z_{t}\right)$ and $\mathcal{L}\left(z_{t}\right) \cap \mathcal{L}^{c}(\overrightarrow{0})$. By Lemma 3.3.2 and Theorem 3.3.3 we have

$$
\mathcal{L}(\overrightarrow{0}) \cap \mathcal{L}^{c}\left(z_{t}\right)=\left\{x \in \Gamma_{7} \mid\left(x \mid z_{t}\right)=1\right\} .
$$

Now $\mathcal{L}\left(z_{t}\right) \cap \mathcal{L}^{c}(\overrightarrow{0})=z_{t} \oplus\left(\mathcal{L}(\overrightarrow{0}) \cap \mathcal{L}^{c}\left(z_{t}\right)\right)$. Hence by $(7), y \in \mathcal{L}\left(z_{t}\right) \backslash \mathcal{L}(\overrightarrow{0}) \Longleftrightarrow$ $y \oplus z_{t} \in \Gamma_{7}$ and $\left(y \mid z_{t}\right)=\left(y \oplus z_{t} \mid z_{t}\right)=1$. Now if $\left(y \mid z_{t}\right)=1$, then since $z_{t} \in \Gamma_{7}$ we have $y \oplus z_{t} \in \Gamma_{7}$ if and only if $y \notin \Gamma_{7}$. Thus we see that

$$
\mathcal{L}\left(z_{t}\right) \cap \mathcal{L}^{c}(\overrightarrow{0})=\left\{y \in V \mid y \notin \Gamma_{7},\left(y \mid z_{t}\right)=1\right\} .
$$

By Proposition 3.3.4(i), the set $\Gamma_{s}$ is characterized by

$$
y \in \Gamma_{s} \quad \Longleftrightarrow \quad\left(y \mid z_{i}\right)= \begin{cases}1 & \text { if } i=s \\ 0 & \text { if } i>s .\end{cases}
$$


Thus, using (8) we see that

$$
\begin{aligned}
\Gamma_{s} & =\left\{y \in \Gamma \mid y \notin \Gamma_{7}, \quad\left(y \mid z_{s}\right)=1,\left(y \mid z_{t}\right)=0 \text { for all } t>s\right\} \\
& =\left\{y \in \Gamma \mid y \notin \Gamma_{7}, \quad\left(y \mid z_{s}\right)=1\right\} \backslash \bigcup_{t=s^{\prime}}^{7}\left\{y \in \Gamma \mid y \notin \Gamma_{7}, \quad\left(y \mid z_{t}\right)=1\right\} \\
& =\left(\mathcal{L}\left(z_{s}\right) \cap \mathcal{L}^{c}(\overrightarrow{0})\right) \backslash\left(\bigcup_{t=s^{\prime}}^{7} \mathcal{L}\left(z_{t}\right) \cap \mathcal{L}^{c}(\overrightarrow{0})\right) \\
& =\left(\mathcal{L}\left(z_{s}\right) \cap \mathcal{L}^{c}\left(z_{7}\right)\right) \backslash\left(\bigcup_{t=s^{\prime}}^{7} \mathcal{L}\left(z_{t}\right) \cap \mathcal{L}^{c}\left(z_{7}\right)\right) \\
& =\mathcal{L}\left(z_{s}\right) \cap\left(\bigcap_{7 \geq t>s} \mathcal{L}^{c}\left(z_{t}\right)\right) .
\end{aligned}
$$

On the other hand, using (7) and the fact that $\left(z_{s} \mid z_{t}\right)=1$ for $t \neq s$,

$$
\begin{aligned}
\Gamma_{s} \oplus z_{s} & =\left\{x \in \Gamma_{7} \mid\left(x \oplus z_{s} \mid z_{s}\right)=1,\left(x \oplus z_{s} \mid z_{t}\right)=0 \text { for all } t>s\right\} \\
& =\left\{x \in \Gamma_{7} \mid\left(x \mid z_{t}\right)=1, \text { for all } t \geq s\right\} \\
& =\bigcap_{7>t \geq s} \mathcal{L}(\overrightarrow{0}) \cap \mathcal{L}^{c}\left(z_{t}\right) \\
& =\mathcal{L}(\overrightarrow{0}) \cap\left(\bigcap_{7>t \geq s} \mathcal{L}^{c}\left(z_{t}\right)\right)
\end{aligned}
$$

\subsection{Partial ordering}

We now show how one can recover the partial ordering on $\Delta_{s}^{+}$from $\Gamma_{s}$.

Lemma 3.4.1. Let $x_{1}, x_{2} \in \Gamma_{7}$. If $x_{1}$ and $x_{2}$ are orthogonal, there exists a unique vector $x_{3} \in \Gamma_{7}$ such that $\left\{x_{1}, x_{2}, x_{3}\right\}$ are pairwise orthogonal, and moreover, $x_{1} \oplus x_{2} \oplus x_{3}=\overrightarrow{0}$. Conversely, if $x_{1} \oplus x_{2} \in \Gamma_{7}$ then $x_{1}$ and $x_{2}$ are orthogonal.

In light of Theorem 3.3.3, this is quite easy to show for our preferred choice of $S$. Nevertheless, this result is true for any $S$ satisfying (1), and so we give a more general proof.

Proof. Let $\beta_{1}=f^{-1}\left(x_{1}\right) \in \Delta_{7}^{+}$and $\beta_{2}=f^{-1}\left(x_{2}\right) \in \Delta_{7}^{+}$. View $\beta_{1}$ and $\beta_{2}$ as roots in the $E_{8}$ root system. Assume that $x_{1}$ and $x_{2}$ are orthogonal; hence $\left\langle\beta_{1}, \beta_{2}\right\rangle=0$.

To begin, for any $\beta \in \Delta_{7}^{+}$, we have $\left\langle\alpha_{8}, \beta\right\rangle=-1$ so $\alpha_{8}+\beta$ is a root of $E_{8}$. Similarly, $\left\langle\alpha_{8}+\beta_{1}, \beta_{2}\right\rangle=-1$ so $\alpha_{8}+\beta_{1}+\beta_{2}$ is a root of $E_{8}$.

To show existence, let $\hat{\alpha}_{8}$ denote the affine (lowest) root of $E_{8}$, which has the property that $\left\langle\hat{\alpha}_{8}, \gamma\right\rangle=0$ for all roots $\gamma \in \Delta\left(E_{7}\right)$. Then $\left\langle\alpha_{8}+\beta_{1}+\beta_{2}, \alpha_{8}+\hat{\alpha}_{8}\right\rangle=-1$ so $\hat{\alpha}_{8}+2 \alpha_{8}+$ $\beta_{1}+\beta_{2}$ is a root. Let

$$
\beta_{3}=-\left(\hat{\alpha}_{8}+2 \alpha_{8}+\beta_{1}+\beta_{2}\right)
$$


and $x_{3}=f\left(\beta_{3}\right)$. Note that $\beta_{3}^{8}=0, \beta_{3}^{7}=1$, so $\beta_{3} \in \Delta_{7}^{+}$, hence $x_{3} \in \Gamma_{7}$. And we can explicitly check $\left\langle\beta_{3}, \beta_{1}\right\rangle=\left\langle\beta_{3}, \beta_{2}\right\rangle=0$, so $\left\{x_{1}, x_{2}, x_{3}\right\}$ are pairwise orthogonal. Thus, for all $\gamma \in \Delta\left(E_{7}\right)$, we have

$$
\begin{aligned}
\left(x_{1} \oplus x_{2} \oplus x_{3} \mid f(\gamma)\right) & =\left\langle\beta_{1}+\beta_{2}+\beta_{3}, \gamma\right\rangle \quad(\bmod 2) \\
& =\left\langle\hat{\alpha}_{8}-2 \alpha_{8}, \gamma\right\rangle \quad(\bmod 2) \\
& =0 .
\end{aligned}
$$

Thus $x_{1} \oplus x_{2} \oplus x_{3}=\overrightarrow{0}$.

For uniqueness, let $x_{3}$ be any vector orthogonal to $x_{1}$ and $x_{2}$, and let $\beta_{3}=f^{-1}\left(x_{3}\right) \in$ $\Delta_{7}^{+}$. We have $\left\langle\alpha_{8}+\beta_{1}+\beta_{2}, \alpha_{8}+\beta_{3}\right\rangle=-1$, so $\gamma=-\left(2 \alpha_{8}+\beta_{1}+\beta_{2}+\beta_{3}\right)$ is a root of $E_{8}$. But $(\gamma)^{8}=-2$, and the only root of $E_{8}$ with this property is the affine root $\hat{\alpha}_{8}$, since $\hat{\alpha}_{8}+\alpha_{8} \succ \hat{\alpha}$ is the unique element covering the affine root and already satisfies $\left(\hat{\alpha}_{8}+\alpha_{8}\right)^{8}>-2$. We conclude that (9) must hold.

For the converse, note that if $x_{1}$ and $x_{2}$ are not orthogonal, then $\beta_{1}-\beta_{2}$ is a root not in $\Delta_{7}$, hence $x_{1} \oplus x_{2} \notin \Gamma_{7}$.

Corollary 3.4.2. Suppose $x, y \in \Gamma_{s}$. Then $(x \mid y)=0$ if and only if $x \oplus y \in \Gamma_{7}$.

Proof. By Proposition 3.3.4(ii), $x \oplus z_{s}, y \oplus z_{s} \in \Gamma_{7}$. Thus $(x \mid y)=0 \Longleftrightarrow\left(x \oplus z_{s} \mid y \oplus z_{s}\right)=0$ $\Longleftrightarrow x \oplus y=\left(x \oplus z_{s}\right) \oplus\left(y \oplus z_{s}\right) \in \Gamma_{7}$, by Lemma 3.4.1.

Theorem 3.4.3. Let $\alpha \in \Delta_{t}^{+}, \beta \in \Delta_{s}^{+}$with $t<s$. Then $f(\beta) \oplus f(\alpha) \in \Gamma_{s}$ if and only if either $\beta+\alpha \in \Delta_{s}^{+}$or $\beta-\alpha \in \Delta_{s}^{+}$.

Proof. Certainly if one of $\beta \pm \alpha \in \Delta_{s}^{+}$, then $f(\beta) \oplus f(\alpha) \in \Gamma_{s}$. Suppose that neither $\beta+\alpha$ nor $\beta-\alpha$ is in $\Delta_{s}^{+}$. Then in fact, neither is a root, so $\langle\beta, \alpha\rangle=0$. Suppose that $f(\beta) \oplus f(\alpha) \in \Gamma_{s}$. Then

$$
\begin{aligned}
(f(\beta) \oplus f(\alpha) \mid f(\beta)) & =(f(\alpha) \mid f(\beta)) \\
& =\langle\beta, \alpha\rangle \quad(\bmod 2) \\
& =0 .
\end{aligned}
$$

By Corollary 3.4.2, we conclude $f(\alpha) \in \Gamma_{7}$, which is impossible, since $\alpha \in \Delta_{t}^{+}$and $t<s \leq 7$.

One can visualise $\Gamma_{7}$ as the squares one sees looking at the corner of a $3 \times 3 \times 3$ cube. The elements are arranged as shown in Figure 4. We can recover the Hasse diagram $\mathrm{H}_{7}$ of the poset structure on $\Delta_{7}^{+}$in this picture, as follows. For each simple root image $a_{i}=f\left(\alpha_{i}\right), i=1, \ldots, 6$, we draw an edge joining $x$ and $y$ if $y=x \oplus a_{i}$. By Theorem 3.4.3 we will draw such an edge if and only if the corresponding roots in $\Gamma_{7}$ are related by addition of a simple root, which is exactly how the Hasse diagram is constructed. A similar procedure also works on the smaller strata.

In this picture, orthogonality is easy to determine as well. By Theorem 3.3.3 this is determined by the links in $\mathrm{T}(V)$. For any $x \in \Gamma_{7}$, the set of $y \in \Gamma_{7}$ orthogonal to $x$ can 
be described as follows: if $y$ is on the same face of the $3 \times 3 \times 3$ cube as $x$, then $y$ is not in the same row or column as $x$; if $y$ is on a different face from $x$ then $y$ is in the same extended row/column as $x$. Figure 4 shows $\mathcal{L}(021) \cap \Gamma_{7}$, which is the set of root images orthogonal to 021 .
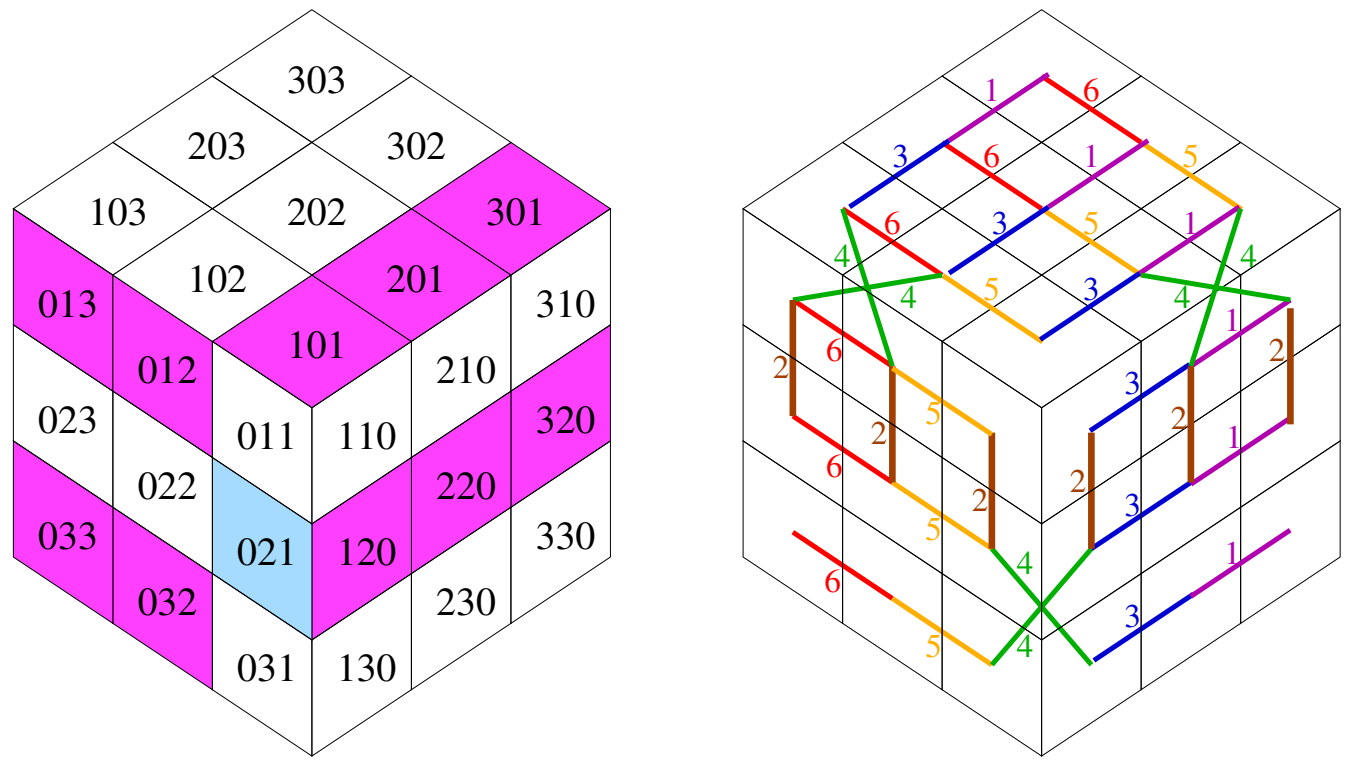

Figure 4: Orthogonality and partial order in $\Gamma_{7}$.

More generally, one can compare $\alpha \in \Delta_{s}^{+}$and $\beta \in \Delta_{t}^{+}$by considering $\tilde{\alpha}$ and $\tilde{\beta}$.

Theorem 3.4.4. Suppose $\alpha \in \Delta_{s}^{+}$and $\beta \in \Delta_{t}^{+}$, and $s \leq t$. Then $\alpha<\beta$ if and only if $\tilde{\alpha}<\tilde{\beta}$. If $s=t$ then $\alpha$ is orthogonal to $\beta$ if and only if $\tilde{\alpha}$ is orthogonal to $\tilde{\beta}$. If $s<t$ then $\alpha$ is orthogonal to $\beta$ if and only if $\tilde{\beta}$ is orthogonal to both $\tilde{\alpha}$ and $\zeta_{s}$, or to neither.

Proof. For the first statement, the 'if' direction is clear, as $\beta-\alpha>\tilde{\beta}-\tilde{\alpha}$. Conversely, if $\beta>\alpha$ then $\beta^{i} \geq 1$ for $i=s^{\prime}, \ldots, t$, hence $\tilde{\beta}-\tilde{\alpha}=\beta-\alpha-\sum_{i=s^{\prime}}^{t} \alpha_{i}$ is still positive.

The statements about orthogonality follow from the following calculation:

$$
(f(\alpha) \mid f(\beta))= \begin{cases}(f(\tilde{\alpha}) \mid f(\tilde{\beta})) & \text { if } s=t \\ (f(\tilde{\alpha}) \mid f(\tilde{\beta})) \oplus\left(z_{s} \mid f(\tilde{\beta})\right) & \text { if } s<t .\end{cases}
$$

The orthogonality and order structures on $\Gamma_{7}$ interact in curious ways, as exemplified by the following question.

Question 3.4.5. For all $x \in \Gamma_{7}$, the poset structure on $\mathcal{L}(x) \cap \Gamma_{7}$ is isomorphic to the partial order on the weights of the 10-dimensional defining representation of $S O(5)$. Why does this happen? 


\subsection{Action of the Weyl group of $E_{6}$ on $\Gamma_{7}$}

The graph $\mathrm{O}\left(\Gamma_{7}\right)$ is a well known object; its complement is the Schläfli graph (see e.g. $[1,3,4]$ for alternate descriptions), which describes the incidence relations of the 27 lines on a cubic surface. It is well known that the full automorphism group of the Schläfli graph is the Weyl group of $E_{6}$. Many of these automorphisms are manifest from our description.

If $\phi_{1}, \phi_{2}, \phi_{3}$ are automorphisms of $F$, then

$$
\left(a_{1}, a_{2}, a_{3}\right) \mapsto\left(\phi_{1}\left(a_{1}\right), \phi_{2}\left(a_{2}\right), \phi_{3}\left(a_{3}\right)\right)
$$

is manifestly an automorphism of the Schläfli graph. If $\pi \in S_{3}$ is a permutation of $\{1,2,3\}$ then we have the automorphism

$$
\left(a_{1}, a_{2}, a_{3}\right) \mapsto\left(a_{\pi(1)}, a_{\pi(2)}, a_{\pi(3)}\right) .
$$

If $\alpha \in \Delta\left(E_{6}\right)$, then the action of the reflection $r_{\alpha}$ on $\Gamma_{7}$ is given by

$$
r_{\alpha}(f(\beta))=f\left(r_{\alpha}(\beta)\right)= \begin{cases}f(\beta \pm \alpha) & \text { if }\langle\alpha, \beta\rangle=\mp 1 \\ f(\beta) & \text { if }\langle\alpha, \beta\rangle=0 .\end{cases}
$$

Using Theorem 3.4.3, we see that for $x \in \Gamma_{7}$,

$$
r_{\alpha}(x)= \begin{cases}x \oplus f(\alpha) & \text { if } x \oplus f(\alpha) \in \Gamma_{7} \\ x & \text { otherwise. }\end{cases}
$$

Each $r_{\alpha}$ swaps six pairs $x \leftrightarrow x \oplus f(\alpha)$ and the restriction of $\mathrm{O}(V)$ to these 12 vertices is a union of two $K_{6}$ graphs, which are maximal cliques. These pairs are known as Schläfli double sixes - there are 36 in total, each arising in this way for some unique $\alpha \in \Delta^{+}\left(E_{6}\right)$.

From (12), it is easy to verify that the automorphisms (10) are generated by reflections in the roots $\alpha_{1}, \alpha_{3}$ (generating all possible $\phi_{1}$ ); $\hat{\alpha}_{6}, \alpha_{2}$ (for $\phi_{2}$ ); $\alpha_{6}, \alpha_{5}$ (for $\phi_{3}$ ); whereas the automorphism (11) corresponds to $S_{3}$ symmetry of the affine Dynkin diagram $\widehat{\operatorname{Dyn}}\left(E_{6}\right)$. These alone do not generate the Weyl group of $E_{6}$; however, together with $r_{\alpha_{4}}$ they do, since this extended list includes all reflections in simple roots.

\subsection{A compression of $E_{6}$}

The discussion in Sections 3.3 and 3.4 gives a description of the strata $\Delta_{s}^{+}$for all $s \leq 7$, but it is not the most symmetrical one for $s \leq 6$. For $s \leq 5$, it is easy to obtain nice description of the strata, as they are subsets of the $D_{5}$ root system. For $s=6$, we can obtain a pleasant description by compressing modulo 3 .

Let $\Delta$ be the $E_{6}$ root system. Let $V=(\mathbb{Z} / 3)^{5}$, with the standard symmetric form (3). Let $S=\left\{a_{1}, \ldots, a_{6}\right\}$, where

$$
\begin{array}{ll}
a_{1}=12000 & a_{2}=00012 \\
a_{3}=01200 & a_{4}=00120 \\
a_{5}=00011 & a_{6}=11111
\end{array}
$$

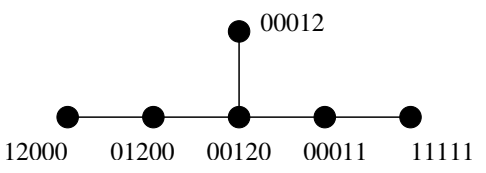


Proposition 3.6.1. For $S$ as above the relations (1) hold.

As a consequence of we obtain the following corollary of Theorem 2.2.3, we have

Corollary 3.6.2. The map $f: \Delta \rightarrow \Gamma$ is a bijection.

Proof. It is an injection by Theorem 2.2.3. To show it is a bijection, we must calculate the size of $\Gamma$. The vectors in $\Gamma$ have either 2 or 5 non-zero coordinates, each of which can be \pm 1 . Thus there are $2^{2}\left(\begin{array}{l}5 \\ 2\end{array}\right)+2^{5}\left(\begin{array}{l}5 \\ 5\end{array}\right)=72$ elements in $\Gamma$. But $\#(\Delta)=72$, so $f$ is a bijection.

Let $\Gamma_{s}=f\left(\Delta_{s}\right)$, and $\Gamma_{s}^{+}=f\left(\Delta_{s}^{+}\right)$denote the images of the strata under $f$.

Theorem 3.6.3. The image of the top stratum, $\Gamma_{6}^{+}$, is the set of vectors in $V$ with all coordinates non-zero, and an even number of coordinates equal to 2:

$$
\Gamma_{6}^{+}=\left\{\left(x_{1}, \ldots, x_{5}\right) \in V \mid \prod_{i=1}^{5} x_{i}=1\right\} .
$$

Thus the elements of $\Gamma_{6}$ are those for which all five coordinates are non-zero, and the elements of $\Gamma \backslash \Gamma_{6}$ are those which have exactly two non-zero coordinates.

Proof. The argument is parallel to the proof of Lemma 3.3.2. The automorphisms of $\Delta\left(E_{6}\right)$ defined in Lemma 3.3.2 restrict to automorphisms of $\Gamma_{6}^{+}$, for similar reasons. As shown in Figure 5, the automorphisms corresponding to Dynkin diagram vertices $v_{3}, v_{4}$ and $v_{5}$ are

$$
\left(x_{1}, x_{2}, x_{3}, x_{4}, x_{5}\right) \mapsto \begin{cases}\left(x_{2}, x_{1}, x_{3}, x_{4}, x_{5}\right) & \text { for } v_{3} \\ \left(x_{3}, x_{2}, x_{1}, x_{5}, x_{4}\right) & \text { for } v_{4} \\ \left(x_{5}, x_{4}, x_{3}, x_{2}, x_{1}\right) & \text { for } v_{5} .\end{cases}
$$

Each of these is a permutation of the symbols coordinates of $V$. If we call these permutations $a, b$ and $c$, respectively, we see that $a, b a b, c b a b c$ and $c a c$ are the standard generators of the symmetric group $S_{5}$. Thus $S_{5}$ acts on the coordinates of $\Gamma_{6}^{+}$. Furthermore, the automorphism corresponding to $v_{6}$ is $\left(x_{1}, x_{2}, x_{3}, x_{4}, x_{5}\right) \mapsto\left(-x_{1},-x_{2},-x_{3},-x_{4}, x_{5}\right)$. Applying these automorphisms to $11111=f\left(\alpha_{6}\right)$, we see that all 16 elements of $\Gamma_{6}^{+}$are indeed of the correct form.

We define $\mathbf{T}(V)$ to be the graph whose vertex set is $V$, and $x=\left(x_{1}, \ldots, x_{5}\right)$ is adjacent to $y=\left(y_{1}, \ldots, y_{5}\right)$ if either $x_{i}=y_{i}$ for exactly one $i$ or $x_{i} \neq y_{i}$ for exactly one $i$. As before, for $X \subset V$, let $\mathrm{T}(X)$ denote the restriction of $\mathrm{T}(V)$ to $X$.

Theorem 3.6.4. The graphs $\mathrm{T}(\Gamma)$ and $\mathrm{O}(\Gamma)$ coincide. Thus $x, y \in \Gamma_{6}^{+}$are orthogonal if and only if $x_{i}=y_{i}$ for exactly one $i$. 


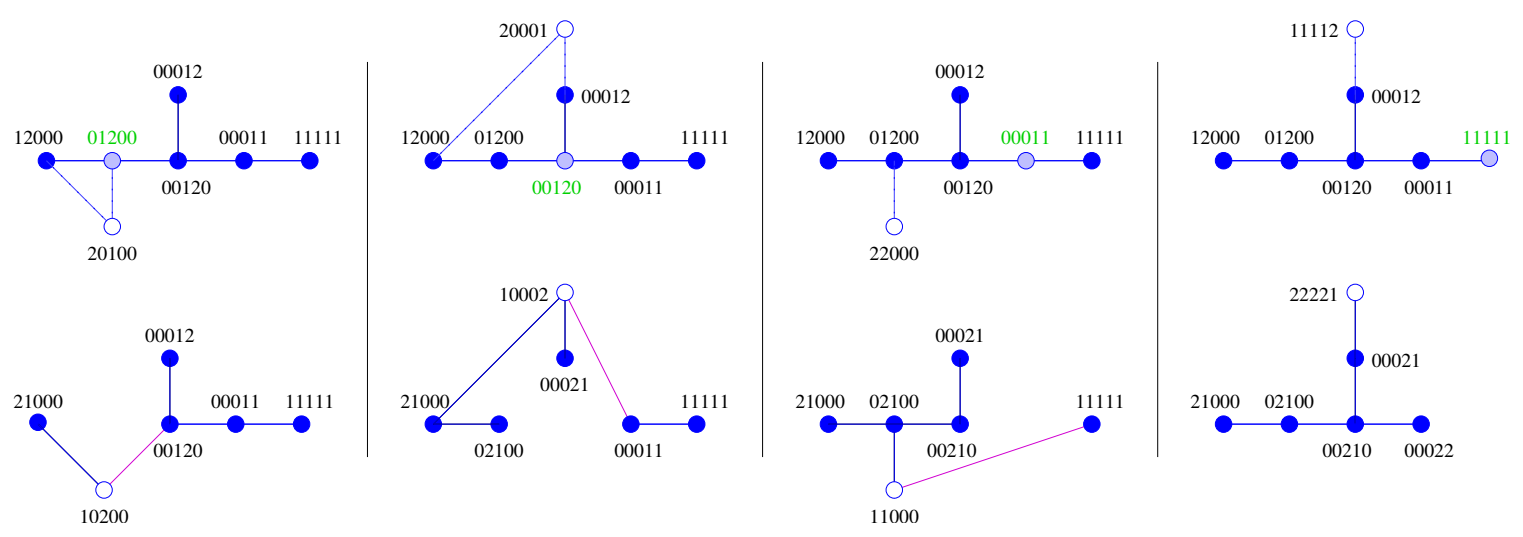

Figure 5: The automorphisms of $\Gamma_{6}^{+}$, corresponding (from left to right) to $E_{6}$ Dynkin diagram vertices $v_{3}, v_{4}, v_{5}$ and $v_{6}$. The top figure is the diagram after Step 1, and the bottom is after Step 4 .

Proof. Suppose $x, y$ are distinct elements of $\Gamma_{6}$. Then $x_{1} y_{1}, x_{2} y_{2}, x_{3} y_{3}, x_{4} y_{4}, x_{5} y_{5} \in\{ \pm 1\}$, and their sum is zero if and only if one of these products is not equal to the other four. Thus $(x \mid y)=0 \Longleftrightarrow x$ and $y$ are adjacent in $\mathrm{T}\left(\Gamma_{6}\right)$.

Suppose $x, y$ are distinct elements of $\Gamma \backslash \Gamma_{6}$. Then exactly two coordinates of $x$ are non-zero, say $x_{i}$ and $x_{i^{\prime}}$, and exactly two coordinates of $y$ are non-zero, say $y_{j}$ and $y_{j^{\prime}}$. If $\left\{i, i^{\prime}\right\} \cap\left\{j, j^{\prime}\right\}=\emptyset$ then $(x \mid y)=0$ and $x$ and $y$ agree in exactly one coordinate. If $\#\left(\left\{i, i^{\prime}\right\} \cap\left\{j, j^{\prime}\right\}\right)=1$ then $(x \mid y) \neq 0$ and $x$ and $y$ agree in two or three coordinates. If $\left\{i, i^{\prime}\right\}=\left\{j, j^{\prime}\right\}$ then $(x \mid y)=0 \Longleftrightarrow x \neq \pm y \Longleftrightarrow x$ and $y$ disagree in exactly one coordinate.

Now suppose $x \in \Gamma_{6}$ and $y \in \Gamma \backslash \Gamma_{6}$. Let $y_{j}$ and $y_{j^{\prime}}$ be the nonzero coordinates of $y$. Then $(x \mid y)=x_{j} y_{j}+x_{j^{\prime}} y_{j^{\prime}}=0 \Longleftrightarrow$ either $x_{j}=y_{j}$ or $x_{j^{\prime}}=y_{j}$, but not both $\Longleftrightarrow x$ and $y$ disagree in exactly one coordinate.

Finally, if $x, y \in \Gamma_{6}^{+}$, by Theorem 3.6.3 we cannot have $x_{i} \neq y_{i}$ for exactly one $i$. Thus $x$ and $y$ are adjacent in $\mathrm{T}\left(\Gamma_{6}^{+}\right)$iff $x_{i}=y_{i}$ for exactly one $i$.

Theorem 3.6.5. Let $\alpha$ be a positive root. Let $\beta \in \Delta_{s}^{+}$. Then $f(\beta)+f(\alpha) \in \Gamma_{s}^{+}$if and only if $\beta+\alpha \in \Delta_{s}^{+}$.

Proof. Certainly if $\beta+\alpha \in \Delta_{s}^{+}$, then $f(\beta)+f(\alpha) \in \Gamma_{s}^{+}$. Suppose that $\beta+\alpha \notin \Delta_{s}^{+}$. If $\beta+\alpha$ is a root, then it belongs to some stratum other than $\Delta_{s}^{+}$, so $f(\beta)+f(\alpha) \notin \Gamma_{s}^{+}$. If $\beta=\alpha$, then $f(\beta)+f(\alpha)=f(-\beta)$ is the image of a negative root, so $f(\beta)+f(\alpha) \notin$ $\Gamma_{s}^{+}$. Otherwise, since $\beta+\alpha$ is not a root, we must have $\langle\beta, \alpha\rangle \in\{0,1\}$. In this case, $\langle\beta+\alpha, \beta+\alpha\rangle=4+2\langle\beta, \alpha\rangle \neq 2(\bmod 3)$, so in fact $f(\beta)+f(\alpha) \notin \Gamma$.

As we did with $\Delta_{7}^{+}$, we can recover the partial order structure on $\Delta_{6}^{+}$(and the smaller strata) using Theorem 3.6.5. If the elements of $\Gamma_{6}^{+}$are arranged as shown in Figure 6, we join $x$ and $y$ if $x-y$ is the image of a simple root. Using Theorem 3.6.4, orthogonality is also easily determined in this picture. Figure 6 shows the example of $\mathcal{L}(11122) \cap \Gamma_{6}^{+}$, which gives the set of root images orthogonal to 11122 . 

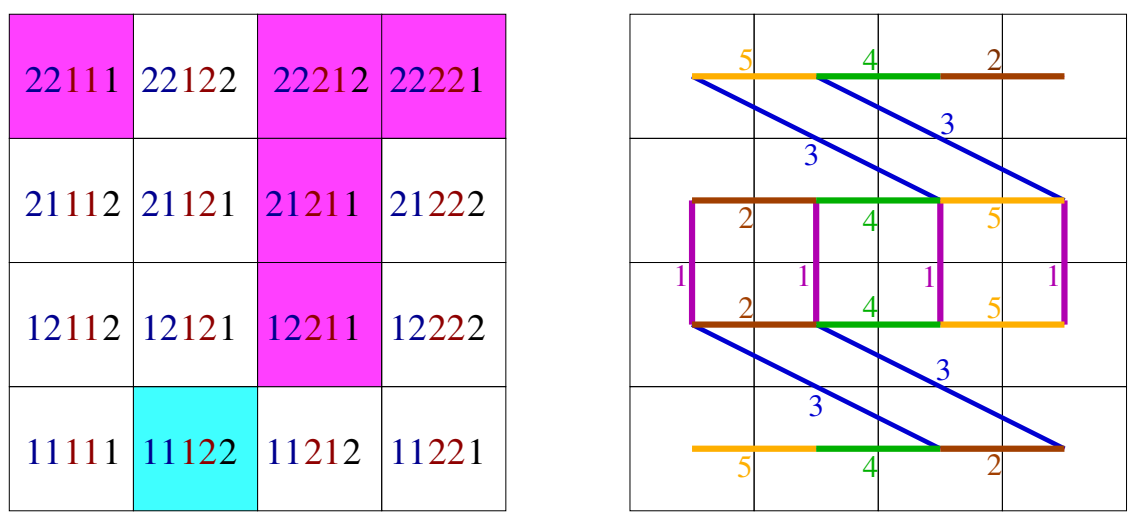

Figure 6: Orthogonality and partial order in $\Gamma_{6}^{+}$.

\section{Order ideals}

\subsection{Relating type $E$ posets through order ideals}

Definition 4.1.1. If $(Y, \leq)$ is a poset, an order ideal in $Y$ is a subset $J \subset Y$ such that if $x \in J$, and $y \leq x$ then $y \in J$. The set of all order ideals in $Y$ is denoted $\mathcal{J}(Y)$ and is itself a poset, ordered by inclusion.

It is a remarkable fact that the posets $\left(\Delta_{s}^{+}, \leq\right)$are related by such a construction: there is an isomorphism

$$
\mathcal{J}\left(\Delta_{s}^{+}\right) \cong \begin{cases}\Delta_{s^{\prime}}^{+} & \text {if } s=3,4,5,6 \\ \Delta_{8}^{+} \backslash\left\{-\hat{\alpha}_{8}\right\} & \text { if } s=7\end{cases}
$$

We refer the reader to [7] for an explanation of this phenomenon.

Definition 4.1.2. If $P$ and $R$ are graphs, an open map from $P$ to $R$ is a function $h: \operatorname{vert}(\mathrm{P}) \rightarrow \operatorname{vert}(\mathrm{R})$ such that

1. $h$ is a homomorphism of graphs, i.e. if $\left(u, u^{\prime}\right) \in \operatorname{edge}(\mathrm{P})$, then $\left(h(u), h\left(u^{\prime}\right)\right) \in$ edge $(R)$;

2. $h$ is locally surjective, i.e. for every $v \in \operatorname{vert}(\mathrm{P}), h$ maps the neighbours of $v$ surjectively to the neighbours of $h(v)$.

Equivalently, $h$ induces an open map on the topological spaces of the graphs.

Proposition 4.1.3. For $3 \leq s \leq 7$, there is a unique function

$$
h_{s}: \Delta_{s}^{+} \rightarrow\{1, \ldots, s\}
$$

such that $h_{s}\left(\alpha_{s}\right)=s$, and $\beta \mapsto v_{h_{s}(\beta)}$ is an open map of graphs from $\mathrm{H}_{s}$ to $\operatorname{Dyn}\left(E_{s}\right)$. For $s=8$ no such function exists. 
The only complete proof we know of this fact is to check it case by case, which is straightforward but unenlightening. We invite the reader to check, for instance, that if one attempts to construct $h_{8}$, it is possible to build the function halfway from both the bottom up and the top down, but the results don't match up. Note that the only "mysterious" part here is uniqueness for $3 \leq s \leq 7$. Existence can be deduced from Proposition 4.1.4, below. Uniqueness can be proven more coherently if one takes an alternate definition for $h_{s}$ (see, e.g. $[10,11]$ ) - the disadvantage in taking such an approach is that we forsake this open map description, which is much easier to work with.

Proposition 4.1.4. The isomorphism (13) is canonical, and given by $\psi_{s}: \mathcal{J}\left(\Delta_{s}^{+}\right) \rightarrow \Delta_{s^{\prime}}^{+}$ where

$$
\psi_{s}(J)=\alpha_{s^{\prime}}+\sum_{\beta \in J} \alpha_{h_{s}(\beta)} .
$$

Proof. It is clear that any isomorphism (13) must be of the form $\psi(J)=\alpha_{s^{\prime}}+\sum_{\beta \in J} \alpha_{h(\beta)}$ for some function $h: \Delta_{s}^{+} \rightarrow\{1, \ldots, s\}$. Since the $\alpha_{s}$ is the minimal element of $\Delta_{s}^{+}$and $\alpha_{s^{\prime}}$ and $\alpha_{s^{\prime}}+\alpha_{s}$ are the two smallest elements of $\Delta_{s^{\prime}}$, we must have $h\left(\alpha_{s}\right)=s$. In light of Proposition 4.1.3, it suffices to show that $h$ must induce an open map from $\mathrm{H}_{s}$ to $\operatorname{Dyn}\left(E_{s}\right)$.

Suppose $\beta \succ \beta^{\prime}$, is an edge of $\mathrm{H}_{s}$, and let $i=h(\beta), i^{\prime}=h\left(\beta^{\prime}\right)$. We show that $\left(v_{i}, v_{i^{\prime}}\right)$ is an edge in the Dynkin diagram, i.e. $\left\langle\alpha_{i}, \alpha_{i^{\prime}}\right\rangle=-1$. Consider the order ideals $J=\left\{\gamma \in \Delta_{s}^{+} \mid \gamma \leq \beta^{\prime}\right\}, J^{\prime}=J \backslash\{\beta\}$ and $J^{\prime \prime}=J \backslash\left\{\beta, \beta^{\prime}\right\}$. We have $\psi(J) \succ \psi\left(J^{\prime}\right) \succ \psi\left(J^{\prime \prime}\right)$, where $\psi(J)-\alpha_{i}=\psi\left(J^{\prime}\right)=\psi\left(J^{\prime \prime}\right)+\alpha_{i^{\prime}}$. Hence $\left\langle\psi(J)-\alpha_{i}, \alpha_{i^{\prime}}\right\rangle=1$. However, note that $\psi(J)-\alpha_{i^{\prime}}$ is not a root. If it were then there would two order ideals between $J$ and $J^{\prime \prime}$, namely $J^{\prime}$ and $\psi^{-1}\left(\psi(J)-\alpha_{i^{\prime}}\right)$, which is impossible if $\beta \succ \beta^{\prime}$. Thus $\left\langle\psi(J), \alpha_{i^{\prime}}\right\rangle \leq 0$. It follows that $\left\langle\alpha_{i}, \alpha_{i^{\prime}}\right\rangle=\left\langle\psi(J), \alpha_{i^{\prime}}\right\rangle-\left\langle\psi(J)-\alpha_{i}, \alpha_{i^{\prime}}\right\rangle \leq-1$. Since $\alpha_{i} \neq-\alpha_{i^{\prime}}$, we must have $\left\langle\alpha_{i}, \alpha_{i^{\prime}}\right\rangle=-1$.

Now, suppose $\beta \in \Delta_{s}^{+}$, and let $i=h(\beta)$. Let $\left(v_{i}, v_{j}\right)$ be an edge in the Dynkin diagram. We show that there exists an edge $(\beta, \gamma) \in \mathrm{H}_{s}$ such that $h(\gamma)=j$.

For every $J \in \mathcal{J}\left(\Delta_{s}^{+}\right)$, let $J^{\prime}=J \backslash\{\beta\}$, and define

$$
\mathcal{J}_{\beta}=\left\{J \in \mathcal{J}\left(\Delta_{s}^{+}\right) \mid J^{\prime} \in \mathcal{J}\left(\Delta_{s}^{+}\right)\right\} .
$$

This set is non-empty: in particular, $J_{\max }=\left\{\beta^{\prime} \in \Delta_{s}^{+} \mid \beta^{\prime} \ngtr \beta\right\}$ is the maximal element of $\mathcal{J}_{\beta}$, and $J_{\min }=\left\{\beta^{\prime} \in \Delta_{s}^{+} \mid \beta^{\prime} \leq \beta\right\}$ is the minimal element.

Consider any pair of order ideals $I, J \in \mathcal{J}_{\beta}$. First note that $\psi(I)-\psi(J) \neq \alpha_{j}$. If this were the case, we would have $\psi\left(J^{\prime}\right)+\alpha_{i}=\psi(J), \psi\left(J^{\prime}\right)+\alpha_{j}=\psi\left(I^{\prime}\right), \psi\left(J^{\prime}\right)+\left(\alpha_{i}+\alpha_{j}\right)=$ $\psi(I)$, and since these are all roots, $\left\langle\psi\left(J^{\prime}\right), \alpha_{i}\right\rangle=\left\langle\psi\left(J^{\prime}\right), \alpha_{j}\right\rangle=\left\langle\psi\left(J^{\prime}\right), \alpha_{i}+\alpha_{j}\right\rangle=-1$, which is impossible. Since the poset $\mathcal{J}_{\beta}$ is connected, it follows that $\psi(I)-\psi(J)$ is a linear combination of simple roots not equal to $\alpha_{j}$. In particular $\psi\left(J_{\max }\right)-\psi\left(J_{\min }\right)$ is a positive linear combination of roots not equal to $\alpha_{j}$. Thus,

$$
\begin{aligned}
\left\langle\psi\left(J_{\max }\right)-\psi\left(J_{\min }^{\prime}\right), \alpha_{j}\right\rangle & =\left\langle\psi\left(J_{\max }\right)-\psi\left(J_{\min }\right), \alpha_{j}\right\rangle+\left\langle\psi\left(J_{\min }\right)-\psi\left(J_{\min }^{\prime}\right), \alpha_{j}\right\rangle \\
& =\left\langle\psi\left(J_{\max }\right)-\psi\left(J_{\min }\right), \alpha_{j}\right\rangle+\left\langle\alpha_{i}, \alpha_{j}\right\rangle \\
& =\left\langle\psi\left(J_{\max }\right)-\psi\left(J_{\min }\right), \alpha_{j}\right\rangle-1 \\
& \leq-1 .
\end{aligned}
$$


We deduce that either $\left\langle\psi\left(J_{\max }\right), \alpha_{j}\right\rangle=-1$ or $\left\langle\psi\left(J_{\min }^{\prime}\right), \alpha_{j}\right\rangle=1$.

In the first case, $\psi\left(J_{\max }\right)+\alpha_{j}$ is a root. Let $K=\psi^{-1}\left(\psi\left(J_{\max }\right)+\alpha_{j}\right)$. We have $K=J_{\max } \sqcup\{\gamma\}$, where $h(\gamma)=j$. By the definition of $J_{\max }$, we must have $\gamma \succ \beta$, so $(\gamma, \beta)$ is an edge of $\mathrm{H}_{s}$. In the second case, $\psi\left(J_{\min }^{\prime}\right)-\alpha_{j}$ is a root. Letting $K=\psi^{-1}\left(\psi\left(J_{\min }^{\prime}\right)-\alpha_{j}\right)$, we have $J_{\text {min }}^{\prime}=K \sqcup\{\gamma\}$, where $h(\gamma)=j$, and $\gamma \prec \beta$, so $(\gamma, \beta)$ is an edge of $\mathrm{H}_{s}$.

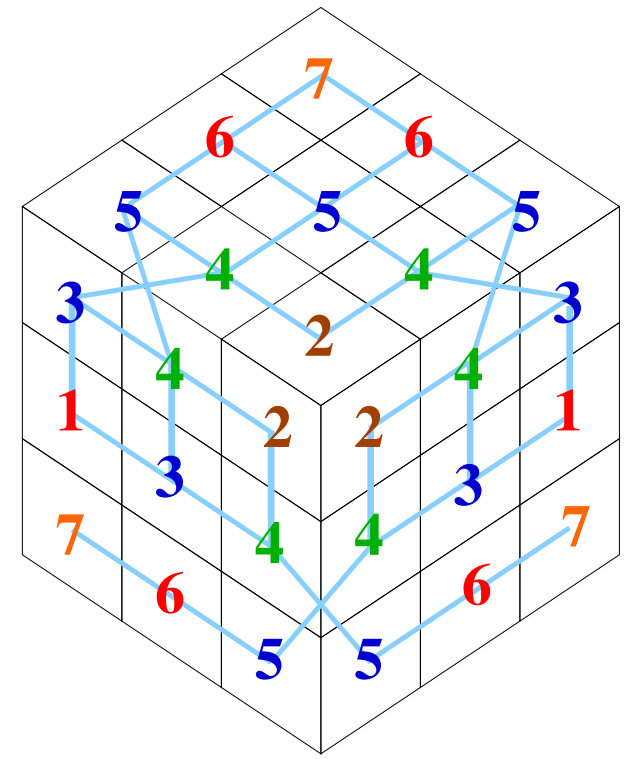

Figure 7: The map $h_{7}$.

The maps $h_{s}$ can be rapidly computed using Proposition 4.1.3. Figure 7 shows $h_{7}$ pictured on the corner of the $3 \times 3 \times 3$ cube. There is a striking symmetry in this picture. If we impose the equivalence relation $1 \sim 6$ and $3 \sim 5$ on $\{1, \ldots, 7\}$, the numbers have full $S_{3}$ symmetry. This equivalence relation comes from the involution on the affine Dynkin diagram $\widehat{\operatorname{Dyn}}\left(E_{7}\right)$. Furthermore the $S_{3}$ symmetry is exactly broken by the rule that $5 \mathrm{~s}$ and $6 \mathrm{~s}$ are connected to a 7 by a path in $\mathrm{H}_{7}$ on the same face of the cube, whereas $1 \mathrm{~s}$ and 3s are not. This symmetry reflects a number of features of the structure of the poset $\Delta_{8}^{+}$; we invite the reader to explore this further.

\section{References}

[1] A.E. Brouwer, A.M. Cohen and A. Neumaier, Distance-regular graphs, SpringerVerlag, Berlin Heidelberg, 1989.

[2] J.H. Conway, R.T. Curtis, S.P. Norton, R.A. Parker and R.A. Wilson, Atlas of finite groups, Oxford University Press, 1985.

[3] P.J. Cameron, Strongly regular graphs, in Selected topics in algebraic graph theory, Cambridge Univ. Press. 
[4] C. Godsil and G. Royle, Algebraic Graph Theory, Springer-Verlag, New York, 2001.

[5] J. Harris, Galois groups of enumerative problems, Duke Math. J. 46 (1979) no. 4, $685-724$.

[6] Yu. Manin, Cubic forms. Algebra, geometry, arithmetic, American Elsevier Publishing Company, New York, 1974.

[7] R.A. Proctor, Bruhat lattices, plane partition generating functions, and minuscule representations, European J. Combin. 5 (1984), no. 4, 331-350.

[8] K. Purbhoo and F. Sottile, The recursive nature of cominuscule Schubert calculus, to appear in Adv. Math.

[9] J.R. Stembridge, On minuscule representations, plane partitions, and involutions in complex Lie groups, Duke Math. J. 73 (1994), 469-490.

[10] N.J. Wildberger, A combinatorial construction for simply-laced Lie algebras, Adv. Appli. Math 30 (2003), 385-396.

[11] N.J. Wildberger, Minuscule posets from neighbourly graph sequences, European J. Cominatorics 24 (2003) 741-757. 